\title{
Barotropic Rossby waves with non-zonal basic flows, topography
}

\author{
and $\delta$ effect
}

\author{
HONGXIN ZHANG ${ }^{1}$ HONGXING CAO ${ }^{2}$ JIAN SONG ${ }^{1}$ \\ 1 College of Sciences, Inner Mongolia University of Technology, Hohhot, 010051 CHINA \\ 756370809@qq.com \\ 2 Chinese Academy of Meteorological Sciences, Beijing,100081 China.caohx@sina.com \\ 1 College of Sciences, Inner Mongolia University of Technology, Hohhot, 010051 CHINA \\ dmwsj@163.com
}

\begin{abstract}
Using the multi-scale method, the stability of barotropic Rossby waves with topography and $\delta$ effects have been studied in non-zonal basic flow. The influences of the non-zonal basic flows and topography and the

parameter $\delta$ on the Rossby waves are discussed. The results show that the W-E oriented topography and the N-S oriented topography on the stability and phase speed of the Rossby waves are quite different; the effect of

parameter $\delta$ is remarkable in the high latitude regions.
\end{abstract}

Key-Words: Rossby waves, non-zonal flows, topography, $\beta$-plane, $\delta$-effect, waves action

Received: August 2, 2019. Revised: April 1, 2020. Accepted: April 20, 2020. Published: April 30, 2020.

\section{Introduction}

There has been an active research and interest over the past years in the solitary of barotropic Rossby waves[1] [2]. The extensive use of the barotropic notably model in operational forecasting has caused a continuing interest in the performance of this model. The Rossby waves provide the simplest model for large scale disturbance in midlatitudes, Durran [3] argued that a simple class of barotropic Rossby waves were shown to propagate westward as the geostrophically balanced meridional wind field periodically reverses in response to a small meridional pressure gradient arising from the latitudinal variation of the Coriolis parameter. Drazin [4] used the linear stability of a parallel zonal flow of an inviscid incompressible fluid on a $\beta$-plane, showing the mode that Rossby waves have been modified by the basic shear was found as the solution of the inverse of a regular Sturm-Liouville problem. Kasahara and Silva Dias[5]studied with a linearized global primitive equation model and confirmed the earlier conclusion of Webster that vertical and horizontal mean shear are needed in order to generate a significant midlatitude response, which is largely equivalent barotropic.

The influence of topography on the atmosphere and ocean, Charney and Eliassen[6], Bolin[7]showed that topography play important role in determining the positions of the Semi-Permanent high-level troughs and ridges in the westerlies, after Charney[8][9]initiated the theory of multiple equilibria, the role of topography has been give more attention. For instance, Paegle[10] showed the effect of topography on Rossby waves; Gottwald and Grimshaw[11] showed the effect of topography on the formation and development of blocking systems. Liu and Tan[12] used barotropic semi-geostrophic mode with topographic forcing the stability and solutions of the nonlinear Rossby waves. The effect of the W-E oriented topography and N-S oriented topography on the stability and phase speed of the 
waves were quite different. $\operatorname{Lv}[13]$ performed the large-scale topography on the stability of Rossby waves, these results showed that the influences of topography with north-south direction slope or west-east direction slope on the Rossby waves and energy propagating were different. Yang[14][15]studied a Rossby wave packet in barotropic flows with asymmetric basic current, topography and $\delta$-effect(the variation of Coriolis parameter with latitude, he explained the $\delta$-effect is capable of describing the structural change of a synoptic disturbance system, the north and south-oriented topography decreased the Rossby wave packet's longitudinal scale and the westward-tilting trough line tilt toward the further eastward, the east-west-oriented topography increased the packet's latitudinal scale. Li[16] studied the baroclinic instability in non-zonal basic flow, the results showed that the appearance of baroclinic instability is more favorable in the non-zonal flows.

In this paper, using the multi-scale method, the stability of barotropic Rossby waves has been studied, It is shown that the influence of topography and $\delta$-effect with non-zonal basic flows on the Rossby waves.

\section{The Governing equation}

The called $\delta$-effect is defined as[14][15]

$$
f \approx f_{0}+\beta_{0}\left(y-y_{0}\right)-\frac{1}{2}\left(y-y_{0}\right)^{2}
$$

in which the Rossby parameter $\beta$ is a linear function of latitude, defined by

$$
\begin{gathered}
\beta=\beta_{0}-\delta_{0}\left(y-y_{0}\right) \\
\beta_{0}=\left.\frac{d f}{d y}\right|_{y=y_{0}} \\
\delta_{0}=\frac{d^{2} f}{d y^{2}} \mid y=y_{0}
\end{gathered}
$$

The propagation of bartropic Rossby waves has been extensively studied by Pedlosky [17], Hoskins and
Karoly [18], Yang[14][15], Luo[19], Cai[20], Schneider[21], and many others. The quasi-geostrophic potential vorticity equation with topography and $\delta$ effect in the barotropic flows be written in the form

$$
\begin{gathered}
\left(\frac{\partial}{\partial t}+\frac{\partial y}{\partial x} \frac{\partial}{\partial y}-\frac{\partial y}{\partial y} \frac{\partial}{\partial x}\right)\left[\nabla^{2} y-\left(B_{0}-\frac{1}{2} d_{0}\left(y-y_{0}\right)\right)\left(y-y_{0}\right)\right] \\
-\frac{f_{0}^{2}}{c_{0}^{2}} \frac{\partial y}{\partial t}-\frac{g f_{0}}{c_{0}^{2}} J(h, y)=0
\end{gathered}
$$

Where the $\psi(x, y, t)$ represents the geostrophic stream function in the barotropic flows, $\nabla^{2}$ is the horizontal Laplace operator, $c_{0}=\sqrt{g H}$ is the shallow water wave speed and is considered to be constant, $h(x, y)$ is the function of topography. Introducing the non-dimensional quantity as follows:

$$
\psi=L U \psi^{*},(X, Y)=L\left(x^{*}, y^{*}\right) t=\frac{L}{U} t^{*}, h=D h^{*}
$$

Where the non-dimensional variables are marked by an asterisk, $L$ is the horizontal scale; $U$ is the horizontal velocity scale and $\frac{L}{U}$ is the advective time scale; $D$ is the depth scale. The non-dimensional form of Equation (5)

$$
\begin{aligned}
& \frac{\partial}{\partial t} \nabla^{2} \psi+\left(\beta_{1}-\delta_{0}\left(y-y_{0}\right)\right) \frac{\partial \psi}{\partial x}-F \frac{\partial \psi}{\partial t} \\
& +J\left(\psi, \nabla^{2} \psi\right)-M J(h, \psi)=0
\end{aligned}
$$

where the asterisk can be dropped for simplicity, $\beta_{1}=\frac{L}{U} \beta_{0} \quad$ (the planetary vorticity factor), $\delta=\frac{L^{3}}{U} \delta_{0}, F=\frac{L^{2}}{L_{0}^{2}}$ (the internal rotational Froude number), $L_{0}^{2}=\frac{c_{0}^{2}}{f_{0}^{2}}$ and $L_{0}$ is the Rossby radius of deformation, $M=\frac{g f_{0}}{c_{0}^{2}} \frac{D L}{U}=\frac{D}{H} \frac{1}{R_{0}}$ is the effect parameter of topography, $R_{0}=\frac{U}{f_{0} L}$ is Rossby 
number. We now assume that the non-dimensional geostrophic stream function consists of an non-zonal flow plus a perturbation stream function

$$
\psi(x, y, t)=\Psi(x, y)+\psi^{\prime}(x, y, t)
$$

Where $\Psi(x, y)=-U_{y}+V_{x}$ is the non-zonal flow[16],

$\mathrm{U}$ is the $\mathrm{x}$-component of basic current, which is a constant; $\mathrm{V}$ is the $\mathrm{y}$-component of basic current, which is too a constant. Inserting (8) into (7) gives an equation

$$
\begin{aligned}
& \left(\frac{\partial}{\partial t}+U \frac{\partial}{\partial x}+V \frac{\partial}{\partial y}\right) \nabla^{2} \psi^{\prime}+B_{0} \frac{\partial \psi^{\prime}}{\partial x}-P_{0} \frac{\partial \psi^{\prime}}{\partial y} \\
& -F \frac{\partial \psi^{\prime}}{\partial t}=-J\left(\psi^{\prime}, \nabla^{2} \psi^{\prime}\right)
\end{aligned}
$$

Where $B_{0}=\beta_{1}-\delta\left(y-y_{0}\right)+M \frac{\partial h}{\partial y}, P_{0}=M \frac{\partial h}{\partial x}$

As in McWilliams[22], Lv[13], Yang[14][15], Luo[19], the Rossby waves of the non-zonal flow and the large-scale topography can be using the multiple scale asymptotic expansion method.

The time and space variables are as follows:

$$
\begin{gathered}
T=\varepsilon t, X=\varepsilon x, Y=\varepsilon y \\
\left.\psi^{\prime}=\varepsilon \psi(10) y, t ; X, Y, T\right)+\varepsilon^{2} \psi_{2}(x, y, t ; X, Y, T)+\ldots
\end{gathered}
$$

where $\epsilon$ is small parameter much smaller than unity $(0<\varepsilon \leq 1)$, it shows the flows are varying slowly in spatial variables and time.

Inserting(10) and (11)into (9) gives the first two order in $\epsilon$ :

$O\left(\varepsilon^{1}\right)$ :

$\left(\frac{\partial}{\partial t}+U \frac{\partial}{\partial x}+V \frac{\partial}{\partial y}\right) \nabla^{2} \psi_{1}^{\prime}+B_{0} \frac{\partial \psi_{1}}{\partial x}-P_{0} \frac{\partial \psi_{1}}{\partial y}-F \frac{\partial \psi_{1}}{\partial t}=0$

$O\left(\varepsilon^{2}\right):$

$$
\begin{gathered}
\left(\frac{\partial}{\partial t}+U \frac{\partial}{\partial x}+V \frac{\partial}{\partial y}\right) \nabla^{2} \psi_{2}^{\prime}+B_{0} \frac{\partial \psi_{2}}{\partial x}-P_{0} \frac{\partial \psi_{2}}{\partial y}-F \frac{\partial \psi_{2}}{\partial t}= \\
-\left[\left(\frac{\partial}{\partial T}+U \frac{\partial}{\partial X}+V \frac{\partial}{\partial Y}\right) \nabla^{2} \psi_{1}+B_{0} \frac{\partial \psi_{1}}{\partial X}-P_{0} \frac{\partial \psi_{1}}{\partial Y}-F \frac{\partial \psi_{1}}{\partial T}\right] \\
{\left[-\left(\frac{\partial}{\partial t}+U \frac{\partial}{\partial x}+V \frac{\partial}{\partial y}\right)\left(\frac{\partial}{\partial x} \frac{\partial \psi_{1}}{\partial X}+\frac{\partial}{\partial y} \frac{\partial \psi_{1}}{\partial Y}+\frac{\partial}{\partial X} \frac{\partial \psi_{1}}{\partial x}\right.\right.} \\
\left.\left.+\frac{\partial}{\partial Y} \frac{\partial \psi_{1}}{\partial y}\right)\right]-J\left(\psi_{1}, \nabla^{2} \psi_{1}\right)
\end{gathered}
$$

Assuming that the spatial and temporal dependence of $\psi_{1}$ may be separated by specifying solution in the form of equation (12), then

$$
\psi_{1}=A(X, Y, T) e^{i \theta}
$$

where $A(X, Y, T)$ is the amplitude wave, $\theta=k x+l y-\omega t$ is the phase function and $\omega, \mathrm{k}, 1$ are, respectively, the angular frequency, the longitudinal wavenumber and the meridional wave number.

Substituting (14) in to (12) obtain the dispersion relation:

$$
\left(k^{2}+l^{2}\right)(\omega-k U-l V)+F \omega+B_{0} k+P_{0} l=0
$$

From the dispersion relation(15), the frequency equation is

$$
\omega=k U+l V-\frac{B k-P l}{K^{2}}
$$

Or

$\omega=\omega_{R}-\frac{\left(F U+M \frac{\partial h}{\partial y}\right) k-\left(M \frac{\partial h}{\partial y}-F V\right) l}{K^{2}}$

Where

$$
\begin{aligned}
& B=B_{0}+F U, P=P_{0}-F V \\
& K^{2}=k^{2}+l^{2}+F, \omega_{R}=U k+V l-\frac{\beta_{1}-\delta_{1}\left(y-y_{0}\right)}{K^{2}} k^{2}
\end{aligned}
$$

is the Rossby waves frequency with $\delta$-effect and non-zonal flows.

(17) shows as follows:

$1 . \delta$-effect and the northern slope of topography $\frac{\partial h}{\partial y}>0$ accelerate moving speed of Rossby wave; 
the zonal flow, $\beta$-effect and the southern slope of topography slow $\frac{\partial h}{\partial y}<0$ moving velocity of Rossby wave, these effects are beneficial to the formation of transverse trough of Rossby wave and to the formation of shear line.

2.For the guided wave trough, the western slope of the topography speeds up the propagation of Rossby waves, the topography of the east slope slows down the propagation of the Rossby waves, and the trough line changes to the north and South directions.

3.For the trailing wave trough, the western slope of the topography slows down the propagation of Rossby waves, and the east slope of topography increases the propagation of Rossby waves, increasing the wavelength of the trailing wave trough, and the trough line will change to the north and South directions.

4.Without consideration beat effect and the parameter, dispersion relation is:

$$
\omega=k U+l V-\frac{\left(F U+M \frac{\partial h}{\partial y}\right) k-\left(M \frac{\partial h}{\partial y}-F V\right) l}{K^{2}}
$$

Equation (18) is the dispersion relation of wave produced by the basic flow shear under the action of topography. When the topography is independent of $\mathrm{X}, \mathrm{Y}$, the wave driven by the non zonal basic shear wave is called vortex wave.

Using (17) obtain the phase velocities

$\mathrm{c}_{x}=\frac{\omega}{k}=U+V \frac{1}{k}-\frac{B-P \frac{1}{k}}{K^{2}}$

$\mathrm{c}_{y}=\frac{\omega}{l}=V+U \frac{k}{1}-\frac{B \frac{k}{1}-P}{K^{2}}$

and $x, y$ components of the group velocities

$\mathrm{c}_{g x}=\frac{\partial \omega}{\partial k}=U-\frac{B}{k^{2}}+\frac{2 k(B k-P l)}{K^{4}}$

$\mathrm{c}_{g y}=\frac{\partial \omega}{\partial l}=V+\frac{P}{k^{2}}+\frac{2 l(B k-P l)}{K^{4}}$

Substitution of (14) into (13) yields the equation of amplitude wave

$$
\begin{aligned}
& \left(\frac{\partial}{\partial T}+U \frac{\partial}{\partial X}+V \frac{\partial}{\partial Y}\right) K^{2} A-(\omega-k U-l V) \\
& {\left[2\left(k \frac{\partial A}{\partial X}+l \frac{\partial A}{\partial Y}\right)+A\left(\frac{\partial k}{\partial X}+\frac{\partial l}{\partial Y}\right)\right]-B \frac{\partial A}{\partial X}+P \frac{\partial A}{\partial Y}=0}
\end{aligned}
$$

\section{The waves action with topography}

\section{and $\delta$-effect}

In the a slowly varying wave train, the wave action which is the ratio of the energy density and the frequency is conserved[23].

Using (20) obtain

$$
\begin{aligned}
& 2 \mathrm{k}(\omega-k U-l V)=-K^{2} \mathrm{c}_{g x}+K^{2} U-B \\
& 2 l(\omega-k U-l V)=-K^{2} \mathrm{c}_{g y}+K^{2} V+P
\end{aligned}
$$

Substitution of (22), (23) into (21),

$$
\begin{aligned}
& K^{2} \frac{D_{g} A}{D T}+\left(\frac{\partial K^{2}}{\partial T}+U \frac{\partial K^{2}}{\partial X}+V \frac{\partial K^{2}}{\partial Y}\right) A \\
& -(\omega-k U-l V)\left(\frac{\partial k}{\partial X}+\frac{\partial l}{\partial Y}\right) A=0
\end{aligned}
$$

Where $\frac{D_{g}}{D T}=\frac{\partial}{\partial T}+c_{g x} \frac{\partial}{\partial X}+c_{g y} \frac{\partial}{\partial Y} \quad$ denotes a derivative following a wave packet.

Add $\frac{\partial(22)}{\partial X}$ to $\frac{\partial(23)}{\partial X}$ obtain

$(\omega-k U-l V)\left(\frac{\partial k}{\partial X}+\frac{\partial l}{\partial Y}\right)=$

$\left(\frac{\partial K^{2}}{\partial T}+U \frac{\partial K^{2}}{\partial X}+V \frac{\partial K^{2}}{\partial Y}\right)-\frac{K^{2}}{2}\left(\frac{\partial c_{g x}}{\partial X}+\frac{\partial c_{g y}}{\partial Y}\right)-\frac{1}{2} \frac{D_{g} K^{2}}{D T}$

Substituting (25) into (24)

$K^{2} \frac{D_{g} A}{D T}+\frac{A K^{2}}{2}\left(\frac{\partial c_{g x}}{\partial X}+\frac{\partial c_{g y}}{\partial Y}\right)+\frac{A}{2} \frac{D_{g} K^{2}}{D T}$

Equation (26) multiplied $\frac{A K^{2}}{B k-P l}$ and defining the generalized wave action density I as following,

$$
I=\frac{\left(A K^{2}\right)^{2}}{4(B k-P l)}
$$




$$
\begin{aligned}
& \left(\frac{\partial I(B k-P l)}{\partial T}+\frac{\partial c_{g x} I(B k-P l)}{\partial X}+\frac{\partial c_{g y} I(B k-P l)}{\partial Y}\right) \\
& =\frac{A^{2}}{2}\left[k^{2} \frac{\partial B}{\partial X}-l^{2} \frac{\partial P}{\partial Y}+k l\left(\frac{\partial B}{\partial Y}-\frac{\partial P}{\partial X}\right)\right]
\end{aligned}
$$

Equation (28) is wave action equation with topography and $\delta$-effect, it is not conserved. Integrating (28) in the considered region, and the perturbation is zero on the boundary,

$$
\iint_{\sigma} \frac{A^{2}}{2}\left[k^{2} \frac{\partial B}{\partial X}-l^{2} \frac{\partial P}{\partial Y}+k l\left(\frac{\partial B}{\partial Y}-\frac{\partial P}{\partial X}\right)\right] d \sigma=0
$$

If $\frac{\partial h}{\partial y}=0$ (29) formula is

$$
\iint_{\sigma} \frac{A^{2}}{2} k l \frac{\partial B}{\partial Y} d \sigma=0
$$

For perturbations of amplitude and wavelength with time, there is (30) the necessary condition for perturbation instability to be at least one zero point in the $\sigma$ region, i.e.

$$
\frac{\partial B}{\partial Y}=M \frac{d^{2} h}{d y^{2}}-\delta=0 . y=y_{d}
$$

Equation(31) shows that topography and $\delta$-effect are the main factors of perturbation instability under the action of non-zonal basic flow.

$$
\begin{gathered}
E=\frac{(K A)^{2}}{4} \\
\frac{\partial E}{\partial T}+\frac{\partial}{\partial X}\left(c_{g x} E\right)+\frac{\partial}{\partial Y}\left(c_{g y} E\right)=0
\end{gathered}
$$

\section{Concluding remarks}

These solitary Rossby waves of the two layer fluid are described by the inhomogeneous $\mathrm{KdV}$ or $\mathrm{mKdV}$ equation depending on the baroclinicity fluid, when the change of $\beta$ and the fluid with the topography. In the general case where the basic flow has the shear, Rossby solitary waves are described by the $\mathrm{KdV}(\mathrm{mKdV})$ equation, but, if considering the change of $\beta$, the Rossby solitary can exit in the absence of horizontal shear in the basic flow. The inhomogeneous terms of the $\mathrm{KdV}$ and $\mathrm{mKdV}$ equation are induce by the bottom topography effect. The internal rotational Froude number $F$ has a certain effect on the Rossby solitary waves steepness, with the decrease of $F$, the solitary waves steepness is increase. There is no effect on the basic flow pattern of solitary waves. The horizontal shear and the vertical shear of the basic flow are also the factor causing solitary waves steepness increase. Finally, the further modifications the Rossby waves will be considered; the instability of the Rossby solitary with the change $\beta$; the Rossby waves in the n-level model. Acknowledgment: The authors thank two anonymous reviewers. This study were supported by the National Natural Science Foundation of China [grant number 11362012]; [grant number 11562014]; [grant number 41465002]; [grant number 41765004] and the High School Science Research Project of the Inner Mongolia Autonomous Region[grant number NJZY16096].

\section{References:}

[1] Kuo H. L. Dynamic instability of two-dimensional nondivergent flow in a barotropic atmosphere. J. Met., 6, 105-122.

[2] E. Eliasen. Numerical solutions of the perturbation equation for linear flow. Tellus, 6 , 183-192.

[3] Dale R. Durran, On a physical mechanism for Rossby wave propagation. J. Atmos. Sci., 45, 4020-4022.

[4] Drazin P. G., D. N. Beaumont and S. A. Coaker, 1982: On Rossby waves modified by basic shear, and barotropic instability. J. Fluid Mech.,124, 439-456.

[5] Kasahara, A., and P. L. Silva Dias, Response of planetary waves to stationary tropical heating in a global atmosphere with meridional and vertical shear. J. Atmos. Sci., 43, 1893-1911.

[6] Charney, J. G., and A. Eliassen. A numerical method for predicting the perturbations of the middle latitude westerlies. Tellus, 1, 38-54.

[7] Bolin, B. On the influence of the earth's orography on the general character of the westerlies. Tellus, 2,184-195. 
[8] Charney, J. G., and J. G. DeVore, Multiple flow equilibria in the atmosphere and blocking. J. Atmos. Sci., 36, 1205-1216.

[9] Charney, J. G., and D. Straus, From-drag instability, multiple equilibria and propagating planetary waves in baroclinic, graphically force, planetary waves systems J. Atmos. Sci., 37, 1157-1176.

[10] Paegle, J. N. The effect of topography on a Rossby wave. J.Atmos.Sci., 36, 2267-2271.

[11] Gottwald, G. and Grimsaw, R.The effect of topography on the dynamics of interacting solitary waves in the context of atmospheric blocking. J. Atmos. Sci, 56, 3663-3678

[12] Liu, S. K., and Tan, B. K. Nolinear Rossby waves Forced by topography. Appl.Math.Mech. 9 229-240(in Chinese).

[13] Lv, K. L. The influences of large orography on instability of Rossby waves in shear currents. Acta Met.Sin. 44 275-281(in Chinese).

[14] Yang, H. J. Evolution of a Rossby wave packet in barotropic flows with asymmetric basic current, topography and $\delta$-effect. J.Atmos.Sci., 44, 2267-2276.
[15] Yang, H. J. Global behavior of the evolution of a Rossby wave packet in barotropic on the earth's $\delta$-surface. J.Atmos.Sci., 45, 133-146.

[16] Li, C. Y. The baroclinic instability in non-zonal basic flows. Acta Met. Sin., 42(in Chinese) 148-135.

[17] Pedlosky.J. Geophysical Fluid Dynamics. Springer-Verlag, 624pp.

[18] Hoskins, G. J., and D. J. Karoly. The steady linear response of a spherical atmosphere to thermal and orographic forcing. J. Atmos. Sci., 38, 1179-1196.

[19] Luo, D. H.A barotropic envelope Rossby soliton model for block-eddy interaction Part I: effect of topography. J.Atmos.Sci., 62, 5-21.

[20] Cai, M. and Huang B. H. A new look at the physics of Rossby waves: a mechanical Coriolis oscillation. J. Atmos Sci., 70, 303-316.

[21] E. K. Schneider. Trajectory analysis of mechanism for westward propagation of Rossby waves. J.Atmos.Sci., 72, 2178-2182.

[22] McWilliams, J. C. Large-scale inhomogeneities and mesoscale ocean waves: a single, stable wave field. J.Mar.Res., 34, 432-456.

[23] Whitham, G. B. Linear and nonlinear wave. John Wile Sons, 374-379pp 